Lau, Stephan ; Haueisen, Jens; Schukat-Talamazzini, Ernst G.; Voss, Andreas;

Görnig, Matthias; Leder, Uwe; Figulla, Hans-R.:

\title{
Low HRV entropy is strongly associated with myocardial infarction
}

Zuerst erschienen in: Biomedizinische Technik = Biomedical Engineering. - Berlin [u.a.] : de Gruyter. - 51 (2006), 4, p. 186-189.

Erstveröffentlichung: $\quad$ 2006-10-25

ISSN (online): $\quad$ 1862-278X

ISSN (print): $\quad$ 0013-5585

DOI: $\quad$ 10.1515/BMT.2006.033

[Zuletzt gesehen: 43696]

„Im Rahmen der hochschulweiten Open-Access-Strategie für die Zweitveröffentlichung identifiziert durch die Universitätsbibliothek IImenau. “

"Within the academic Open Access Strategy identified for deposition by Ilmenau University Library."

„Dieser Beitrag ist mit Zustimmung des Rechteinhabers aufgrund einer (DFGgeförderten) Allianz- bzw. Nationallizenz frei zugänglich."

"This publication is with permission of the rights owner freely accessible due to an Alliance licence and a national licence (funded by the DFG, German

Research Foundation) respectively." 


\section{Low HRV entropy is strongly associated with myocardial infarction}

\author{
Stephan Lau',2, Jens Haueisen,4,*, Ernst G. \\ Schukat-Talamazzini ${ }^{1}$, Andreas Voss ${ }^{3}$, Matthias \\ Goernig $^{5}$, Uwe Leder ${ }^{5}$ and Hans-R. Figulla \\ ${ }^{1}$ Institute of Computer Science, Friedrich Schiller \\ University Jena, Jena, Germany \\ 2 Biomagnetic Center Jena, Friedrich Schiller University \\ Jena, Jena, Germany \\ ${ }^{3}$ Department of Medical Engineering, University of \\ Applied Sciences Jena, Jena, Germany \\ ${ }^{4}$ Institute of Biomedical Engineering and Informatics, \\ Technical University of IImenau, IImenau, Germany \\ ${ }^{5}$ Clinic of Internal Medicine I, Friedrich Schiller \\ University Jena, Jena, Germany
}

\begin{abstract}
Heart rate variability (HRV) is a marker of autonomous activity in the heart. An important application of HRV measures is the stratification of mortality risk after myocardial infarction. Our hypothesis is that the information entropy of HRV, a non-linear approach, is a suitable measure for this assessment. As a first step, to evaluate the effect of myocardial infarction on the entropy, we compared the entropy to standard HRV parameters. The entropy was estimated by compressing the tachogram with Bzip2. For univariate comparison, statistical tests were used. Multivariate analysis was carried out using automatically generated decision trees. The classification rate and the simplicity of the decision trees were the two evaluation criteria. The findings support our hypothesis. The meanNN-normalized entropy is reduced in patients with myocardial infarction with very high significance. One entropy parameter alone exceeds the discrimination strength of multivariate standards-based trees.
\end{abstract}

Keywords: Burrows-Wheeler compression; Bzip2; decision tree; entropy estimation; heart rate variability.

\section{Introduction}

The autonomous nervous system mediates a number of control mechanisms such as oxygen and carbon dioxide concentrations and blood pressure. Heart rate variability (HRV) is a marker of the activity of the autonomous nervous system. In particular, following myocardial infarction, HRV is reduced, an observation that is used to stratify patient risk [1].

\footnotetext{
${ }^{*}$ Corresponding author: Jens Haueisen, Biomagnetic Center Jena, Friedrich Schiller University Jena, Erlanger Allee 101, 07747 Jena, Germany

Phone: + 49-3641-9325770

Fax: +49-3641-9325772

E-mail: haueisen@biomag.uni-jena.de
}

The basic signal used in HRV analysis is the tachogram, which demonstrates the consecutive sequence of beat-to-beat intervals, also called normal-to-normal (NN) intervals. Figure 1 shows the difference between the tachogram of a healthy individual and a patient with recent myocardial infarction.

\section{Standard measures}

The most widely used HRV measures [2] are from the time and frequency domains, such as those in Table 1. However, non-linear HRV methods represent a promising area for research because the abstract concept of variability can be modeled more effectively than just with signal-centered measures and counts.

\section{Information entropy}

Information entropy is such a non-linear method. In information theory, the entropy of a message is a measure of the amount of information or complexity contained in it. A random signal would have large entropy, while a constant signal has small entropy. The more order or repetition or redundancy is in a signal, the lower is its entropy.

\section{Hypothesis and objective}

The concept of HRV is very similar to that of entropy. A healthy tachogram (Figure 1) with considerable variability is unordered and non-repetitive; its entropy is high. A pathological tachogram with low variability has lower entropy. The hypothesis adopted here is that the entropy of the tachogram is an effective measure of HRV.

The primary objective was to test this hypothesis in patients with myocardial infarction. The univariate and multivariate discrimination strength between patients and controls was compared to one of the traditional HRV measures. A secondary objective was to identify the signal preprocessing steps necessary for entropy estimation.

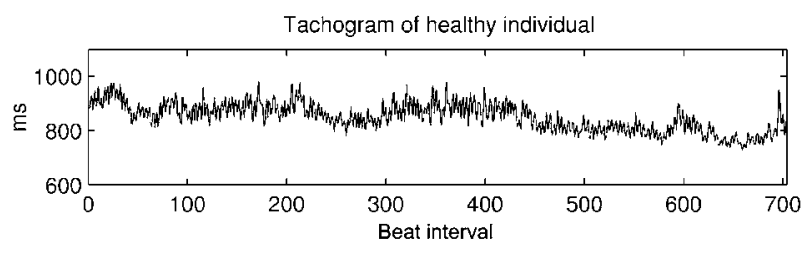

Tachogram of post-infarction patient

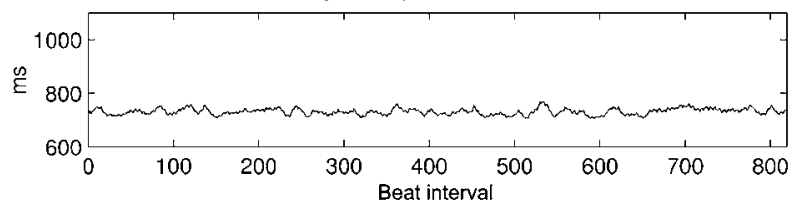

Figure 1 Tachogram of a healthy individual and a post-infarct patient (no diabetes, coronary heart disease degree 3); both were $\approx 50$ years of age and female. 
Table 1 Time- and frequency-domain measures.

\begin{tabular}{ll}
\hline Parameter & Description \\
\hline meanNN & Mean of NN intervals \\
sdNN & Standard deviation of all NN intervals \\
cVNN & Ratio sdNN/meanNN \\
sdaNN1 & sdNN averaged over periods of 1 min \\
rmssd & Root of the mean-squared differences of successive NN intervals \\
pNN50 & Proportion of successive interval differences $\geq 50$ ms within all NN intervals \\
pNNi10 (pNNi20) & Proportion of successive interval differences $\leq 10(20)$ ms within all intervals \\
VLF & Power in the very low range $(\leq 0.04 \mathrm{~Hz})$ \\
LF & Power in the low range $(0.04-0.15 \mathrm{~Hz})$ \\
HF & Power in the high range $(0.15-0.4 \mathrm{~Hz})$ \\
$\mathrm{P}$ & Power over the whole range $(0-0.4 \mathrm{~Hz})$ \\
LF/HF & Ratio LF/HF \\
LF/P & Ratio LF/P \\
HF/P & Ratio HF/P \\
\hline
\end{tabular}

\section{Materials and methods}

\section{Data set}

In 114 subjects (Table 2), combined MCG and ECG recordings were obtained in a supine position. Each recording was of 10 min duration with a sampling frequency of $1000 \mathrm{~Hz}$ and demonstrated a sinus rhythm with less than $10 \%$ extrasystoles. Of the 114 recordings, 59 were from patients with myocardial infarction; the remaining 55 were from healthy controls without a history of heart disease. The time between myocardial infarction of patients and the recording was $11.9 \pm 6.9$ days.

\section{Preprocessing}

The NN intervals were extracted using template matching with a QRS complex as template (Figure 2). Artifacts, mainly extrasystoles, were then replaced by a random value matching the adaptive mean and standard deviation [3]. Every recording was reduced in length to that of the shortest. Both the resulting tachogram and its successive differences were used as separate inputs for HRV analysis.

Quantization of the tachogram was an entropy-specific step. Compression-based entropy requires alphabetical symbols as input. The raw intervals falling between 400 and $1400 \mathrm{~ms}$ were resampled at a rate of $128 \mathrm{~Hz}$. This is similar to sorting the intervals into histogram bins of size $1000 \mathrm{~ms} / 128=7.8125 \mathrm{~ms}$. Thus, there were exactly 128 bins (or symbols) in the alphabet.

\section{Entropy definition and estimation}

Shannon's Theorem formally defines entropy: Imagine a message source that has $n$ possible messages with probabilities $p_{1}, \ldots, p_{n}$. Then the entropy of message $i$ is:

$E_{i}=-\log _{2} p_{i} \quad[\mathrm{bit}]$.

This is intuitive because a message with high proba-

Table 2 Population characteristics.

\begin{tabular}{llll}
\hline & Post-infarct & Controls & All \\
\hline Age (mean \pm SD) (years) & $54.0 \pm 9.4$ & $49.3 \pm 11.2$ & $51.7 \pm 10.5$ \\
Gender (M/F) & $42 / 17$ & $27 / 28$ & $69 / 45$ \\
\hline
\end{tabular}

bility will have low entropy and vice versa. The average entropy $E$ of all messages, also called the entropy of the message source, is then:

$E=-\sum_{i=1}^{n} p_{i} \log _{2} p_{i} \quad[$ bit $]$

In this study, each NN interval is considered a message and the sinus node is the source. A tachogram is a sample of the messages that a source emits [4].

There are two principal methods for estimating the entropy. The first is to estimate the probabilities in some

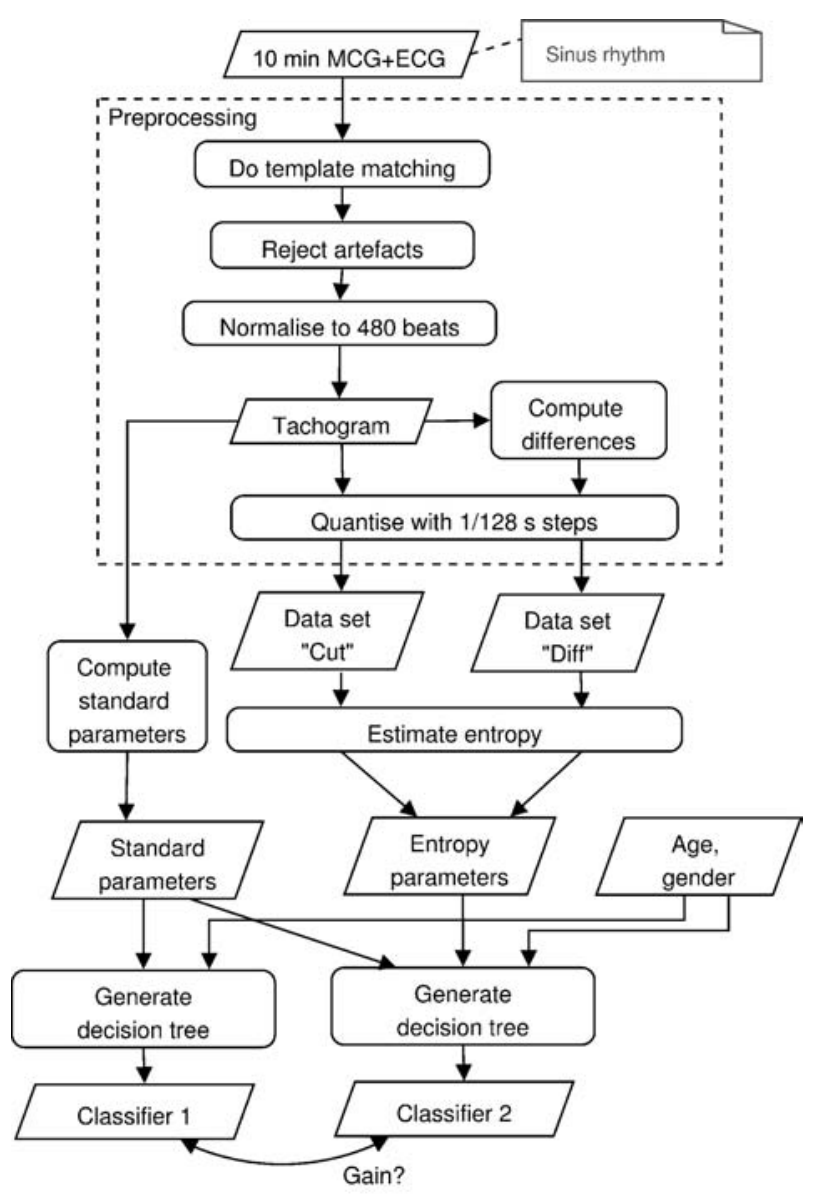

Figure 2 Data processing steps and study design. 
statistical manner, then to compute the entropy directly from this estimate. The second indirect way is to compress the message, thereby eliminating the redundancy contained in it. The compression ratio is then an upper bound of the entropy. The better the compression, the closer is the estimation [4].

In this study, the entropy was estimated by compressing the quantized tachogram with Burrows-Wheeler compression [5], which is one of the most efficient algorithms. Each interval was encoded as 1 byte $=8$ bit in a file. The file was then compressed using Bzip2 [6], an implementation of the Burrows-Wheeler compression. The entropy per bit of the input signal was estimated by:

$E \approx \frac{\text { compressed file size }}{\# \text { of values }{ }^{*} \# \text { of bit per value }} \quad$ [bit].

The compressed file size divided by the number of values, the intervals, yields the entropy per interval. Because each interval was encoded by $7=\log _{2}(128)$ bit during quantization, we normalized by dividing by 7 . The resulting value could then be interpreted as proportion of information in the quantized tachogram.

For the first sample (Figure 1), the entropy was $323 \times 8$ bit $/ 480 \times 7=0.77$ bit $\approx 4 / 5$. However, the post-infarct patient only had entropy of $161 \times 8 \mathrm{bit} / 480 \times 7=0.39 \mathrm{bit}$ $\approx 2 / 5$. The entropy parameters used here were: [BZIP2,Cut], [BZIP2,Diff], [BZIP2,Cut]/m and [BZIP2, Diff]/m. [BZIP2,Cut] means Bzip2 is applied to the tachogram, whereas in [BZIP2,Diff] it is applied to the successive differences. The extension ' $/ \mathrm{m}$ ' means divided by meanNN.

\section{Classification}

Decision trees were chosen as the classification model because they explicitly state the choice of parameters and how they are combined. The decision trees were generated using the CART algorithm [7].

First, a tree was generated and then pruned based on the misclassification costs in a ten-fold cross-validation. To establish the classification rate, leave-one-out validation was performed. These steps were performed for the parameter set in Table 1, in which all parameters except LF/P and HF/P were taken directly from the standard [2], and the augmented set including the entropy. The criteria for assessing the decision trees were: (1) the classification rate, and (2) the simplicity and stability. The
Table 3 Wilcoxon test results.

\begin{tabular}{llll}
\hline Parameter & Male & Female & All \\
\hline meanNN & n.s. & n.s. & 0.03742 \\
sdNN & n.s. & 0.00151 & n.s. \\
cvNN & n.s. & 0.00012 & 0.00331 \\
sdaNN1 & n.s. & 0.00192 & n.s. \\
pNN50 & n.s. & 0.04094 & n.s. \\
VLF & n.s. & 0.01185 & n.s. \\
LF & n.s. & 0.00151 & 0.01522 \\
HF & n.s. & 0.02861 & n.s. \\
P & n.s. & 0.00208 & n.s. \\
LF/P & 0.02330 & n.s. & 0.04121 \\
[BZIP2,Cut] & n.s. & 0.01304 & n.s. \\
[BZIP2,Diff] & n.s. & 0.01862 & n.s. \\
[BZIP2,Cut]/m & 0.01569 & 0.00092 & 0.00004 \\
[BZIP2,Diff]/m & n.s. & 0.00151 & 0.00027 \\
\hline Paramets & with & signfince &
\end{tabular}

Parameters with no significance at all were omitted; n.s., not significant.

best classification rule is the one that represents an effective compromise between both.

\section{Results}

\section{Statistical tests}

Wilcoxon tests (Table 3 ) showed that [BZIP2,Cut] $/ \mathrm{m}$ had the highest significance $(p=0.00004)$ for all recordings and for just men. In women, [BZIP2,Cut]/m was only outperformed by cvNN. [BZIP2,Diff]/m was only slightly less significant than [BZIP2,Cut] $/ \mathrm{m}$ for all and female subjects; for males its $p$-value was 0.0513 . [BZIP2,Cut]/m was the only parameter with significant mean differences in all three groups.

\section{Standards tree}

The tree in Figure 3 shows the most frequent tree during validation and is thus selected as classifier 1 . The classification rate is $65 \%$. During leave-one-out validation, one recording is left out at a time. This produces a different tree each time. The number of leaves varies from 3 to 26, with an average of 5.4. The parameters in Figure 3 are the most frequent ones. Classifier 1 is therefore an unstable classification rule. Additional tests with composite classifiers produced a maximum classification rate of $68 \%$, bearing in mind that these trees are even more complex and unstable.
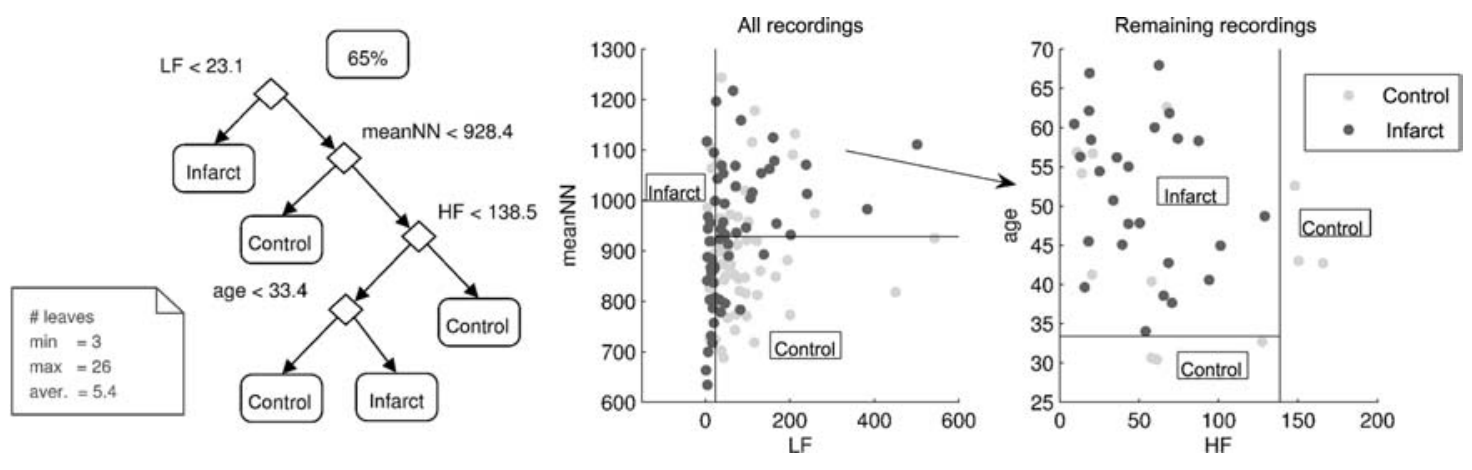

Figure 3 Classifier 1: decision tree based on standard parameters and its visualization. 


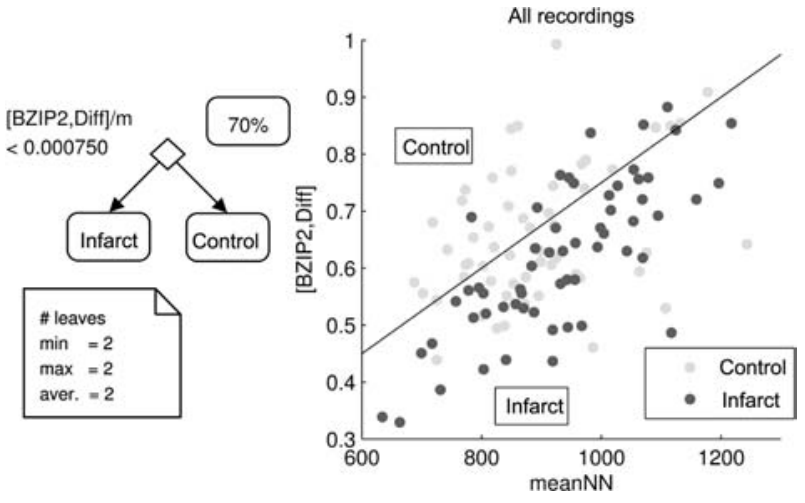

Figure 4 Classifier 2: decision tree based on standard and entropy parameters and its visualization.

\section{Standards and entropy tree}

Augmentation with entropy parameters produces the decision tree in Figure 4. The entropy parameter [BZIP2,Diff]/m supersedes all standard parameters. In fact, this simple [BZIP2,Diff]/m threshold outperforms any multivariate classification rule that uses standard parameters and combinations with entropy parameters. This decision tree has a higher classification rate of $70 \%$. In addition, the very same tree was found in every leaveone-out validation step. This means that this classification rule is much more stable than that of classifier 1. Additional tests with composite classifiers produced a maximum classification rate of $74 \%$.

\section{Discussion}

\section{Standard parameters}

The standard parameters showed discrimination strength in females. In males, only LF/P was significant. The multivariate trees often had $L F$ as the first decision node (Figure 3). A low LF value characterizes infarcts. This complies with the clinical observation that HRV is reduced after infarction [1]. Additional tests with composite trees showed that the combination of meanNN with a parameter such as LF, LF/P or sdNN covers most of the achievable classification rate.

\section{Entropy parameters}

[BZIP2,Cut]/m was the only parameter with discrimination strength in all groups and showed stronger significance than standard parameters. Especially in males, the main patient group, [BZIP2,Cut]/m outperformed the standard parameters. Multivariate analysis identified [BZIP2,Diff] $/ \mathrm{m}$ as the best classification parameter. In the [BZIP2,Diff]/m threshold, the entropy [BZIP2,Diff] is combined with meanNN. This is similar to the parameter $\mathrm{cvNN}=\mathrm{sdNN} /$ meanNN [2]. However, [BZIP2,Diff] captured the variability, not only the variance. The information entropy expressed the variability as a whole.

Entropy fulfilled both of the quality criteria very well, in that it achieved a high classification rate with a simple and stable discrimination rule. This extends the previous findings of Baumert et al. [8] on arrhythmias. An advantage over other entropy-related approaches $[9,10]$ is that the entropy definition is not altered or decomposed. Thus, the original information theory concept of entropy is retained.

In conclusion, we evaluated the usefulness of entropy as an HRV parameter with the long-term goal of risk stratification. In contrast to previous entropy-based approaches, we applied the original information-theoretic definition of entropy. The entropy was estimated by a straightforward compression-based method. To obtain optimal multivariate classification rules, an automated decision tree generator was used. The key findings are:

- $[B Z I P 2, C u t] / m$ is reduced in post-infarction patients with very high significance $(p=0.00004)$.

- The discrimination strength of [BZIP2,Diff]/m alone exceeds that of multivariate standards-based decision trees by $5-10 \%$, while being simpler and more stable.

- The tachogram length required is only $500-600$ beats or $\approx 10-12 \mathrm{~min}$. A suitable quantization step width is $1 / 128 \mathrm{~s}=7.8125 \mathrm{~ms}$.

This confirms the hypothesis that entropy is an effective measure of HRV and highly associated with myocardial infarction. We suggest that the application of BZIP2 entropy is suitable for post-infarct risk stratification.

\section{References}

[1] Kleiger RE, Miller JP, Bigger JT Jr, Moss AJ. Decreased heart rate variability and its association with increased mortality after acute myocardial infarction Am J Cardiol 1987; 59: 256-262.

[2] Task Force of the European Society of Cardiology, the North American Society of Pacing and Electrophysiology. Heart rate variability: standards of measurement, physiological interpretation and clinical use. Eur Heart J 1996; 17: 354-381.

[3] Voss A, Hnatkova K, Wessel N, et al. Multiparametric analysis of heart rate variability used for risk stratification among survivors of acute myocardial infarction. Pace 1998; 21: 186-192.

[4] Bell TC, Cleary JG, Witten IH. Text compression. Advanced reference series on computer science. Englewood Cliffs: Prentice Hall 1990.

[5] Burrows M, Wheeler DJ. A block-sorting lossless data compression algorithm. Technical Report 124. Palo Alto: Digital Systems Research Center 1994.

[6] Seward J. Bzip2 and libbzip2 - a program and library for data compression. Manual. 2001. Available at: www.bzip. org.

[7] Breiman L. Classification and regression trees. New York: Chapman \& Hall 1998.

[8] Baumert M, Baier V, Haueisen J, et al. Forecasting of life threatening arrhythmias using the compression entropy of heart rate. Method Inform Med 2004; 43: 202-206.

[9] Richman JS, Moorman JR. Physiological time-series analysis using approximate entropy and sample entropy. Am J Physiol 2000; 278: 2039-2049.

[10] Costa M, Goldberger AL, Peng CK. Multiscale entropy analysis of biological signals. Phys Rev Lett 2005; 71: 021906. 